\title{
Non-newtonian Rabinowitsch Fluid Effects on the Lubrication Performances of Sine Film Thrust Bearings
}

\author{
Jaw-Ren Lin ${ }^{1}$, , Hsiu-Lu Chiang1, Yu-Kang Chiu ${ }^{2}$ \\ ${ }^{1}$ Department of Mechanical Engineering, Nanya Institute of Technology, Taoyuan, Taiwan \\ ${ }^{2}$ Master Program of Mechanical and Mechatronics Engineering, Nanya Institute of Technology, Taoyuan, Taiwan \\ Email address: \\ jrlin@nanya.edu.tw (Jaw-Ren Lin), rotex@nanya.edu.tw (Hsiu-Lu Chiang), abba0818@gmail.com (Yu-Kang Chiu) \\ ${ }^{*}$ Corresponding author
}

\section{To cite this article:}

Jaw-Ren Lin, Hsiu-Lu Chiang, Yu-Kang Chiu. Non-newtonian Rabinowitsch Fluid Effects on the Lubrication Performances of Sine Film Thrust Bearings. International Journal of Mechanical Engineering and Applications. Vol. 5, No. 3, 2017, pp. 162-167. doi: 10.11648/j.ijmea.20170503.14

Received: March 27, 2017; Accepted: May 15, 2017; Published: May 20, 2017

\begin{abstract}
Thrust bearings are generally developed to sustain axial load generated from rotating machinery. Predicting lubrication performances of thrust bearings plays an important role in engineering applications. Taking into account the effects of non-Newtonian rheology, the lubrication performances for a sine film thrust bearing are investigated in the present paper. By employing a small perturbation technique to the nonlinear non-Newtonian Reynolds equation, analytical solutions of the film pressure, the load-carrying capacity, the friction parameter and the required volume flow rate are derived. Comparing with the case lubricated with a Newtonian lubricant, the non-Newtonian Rabinowitsch fluids (including the pseudo-plastic fluids and the dilatant fluids) show significant influences on the lubrication performances of a sine film thrust bearing, especially for a larger value of the non-Newtonian parameter.
\end{abstract}

Keywords: Non-newtonian Rheology, Sine Film Thrust Bearings, Load Performances

\section{Introduction}

Thrust bearings are generally developed to sustain axial load generated from rotating machinery. Predicting load performances of thrust bearings plays an important role in engineering applications. The load performances of thrust bearings lubricated with a Newtonian lubricant have been investigated by many researchers, for example, the studies by Williams [1], Hamrock [2], Taylor and Dowson [3], Talmage and Carpino [4], Lin et al. [5], and Lin and Hung [6]. Owing to the development of modern engineering, the increasing use of a Newtonian lubricant blended with small amounts of long chained polymer solutions has gained great attention. However, the flow behavior of these kinds of non-Newtonian lubricants displays a nonlinear relationship between the shearing stress and the shearing strain rate. From the experimental works by Wada and Hayashi [7], this nonlinear relationship can be simulated by an empirical cubic stress model, or the so-called Rabinowitsch fluid model. In this non-Newtonian fluid model, the nonlinear relationship for one dimensional flow between the shearing stress $\tau$ and the shearing strain rate $\partial u / \partial y$ holds as the following equation.

$$
\tau+n \tau^{3}=\mu_{0} \frac{\partial u}{\partial y}
$$

In this expression, strain rate $\partial u / \partial y$ denotes the initial viscosity of a Newtonian lubricant, and $n$ represents a nonlinear factor characterizing the non-Newtonian rheology. This non-Newtonian Rabinowitsch fluid model is applicable to dilatant lubricants for $n<0$, to Newtonian lubricants for $n=0$, and to pseudo-plastic lubricants for $n>0$. Many studies have applied this model to investigate the thin film lubrication systems, for example, the squeeze film bearings by Lin [8] and Hsu et al. [9], the hydrostatic bearings by Sharma et al. [10], the journal bearings by Bourging and Gay [11], and the inclined plane slider bearings by Lin [12]. According to their results, the influences of non-Newtonian Rabinowitsch fluids on the lubrication performances of the squeeze film bearings, the hydrostatic bearings, the journal bearings and the inclined plane slider bearings are significantly apparent. Since understanding the lubrication 
performances of thrust bearings is helpful in designing the bearing system. In order to provide more information for the application of non-Newtonian fluids on lubrication fields, the study of non-Newtonian effects of Rabinowitsch fluids on the lubrication performances of sine film thrust bearings is therefore motivated.

According to the Rabinowitsch fluid model, the present paper is to investigate the non-Newtonian effects on the lubrication performances of sine film thrust bearings. By employing a small perturbation technique to the nonlinear non-Newtonian Reynolds equation, analytical expressions of the film pressure, the load capacity, the friction parameter and the required volume flow rate are obtained. Comparing with the case lubricated with a Newtonian lubricant, the lubrication performances of sine film thrust bearings with a non-Newtonian Rabinowitsch fluid are presented and discussed through the variation of the non-Newtonian parameter and the inlet to outlet film ratio.

\section{Analysis}

Figure 1 shows the physical geometry of a sine film thrust bearing lubricated with a non-Newtonian fluid. The bearing length in the $x$-direction is $B_{1}$ and the bearing width perpendicular to the $x z$-plane is $B_{2}$. In the present study, the bearing is considered to be infinitely wide, i.e., $B_{2} \gg B_{1}$. The inlet film height in the $y$-direction is $h_{0}$, the outlet film height is $h_{1}$, and the sliding velocity in the $x$-direction is $V_{s}$. The local film height $h$ for the sine film bearing can be described by

$$
h(x)=h_{1}+h_{\sin }(x)
$$

where the sine curve $h_{\text {sin }}(x)$ can be expressed as:

$$
h_{\sin }(x)=\left(h_{0}-h_{1}\right) \cdot\left[1-\sin \left(\frac{\pi x}{2 B_{1}}\right)\right]
$$

Assume that the thin-film lubrication theory of Williams [1] and Hamrock [2] is applicable, then the incompressible continuity equation and the momentum equations and can be written as follows.

$$
\begin{gathered}
\frac{\partial u}{\partial x}+\frac{\partial v}{\partial y}=0 \\
\frac{\partial \tau}{\partial y}=\frac{\partial p}{\partial x} \\
\frac{\partial p}{\partial y}=0
\end{gathered}
$$

where $u$ and $v$ are the velocity components in the $x$ - and $y$-directions respectively, and $p$ is the film pressure.

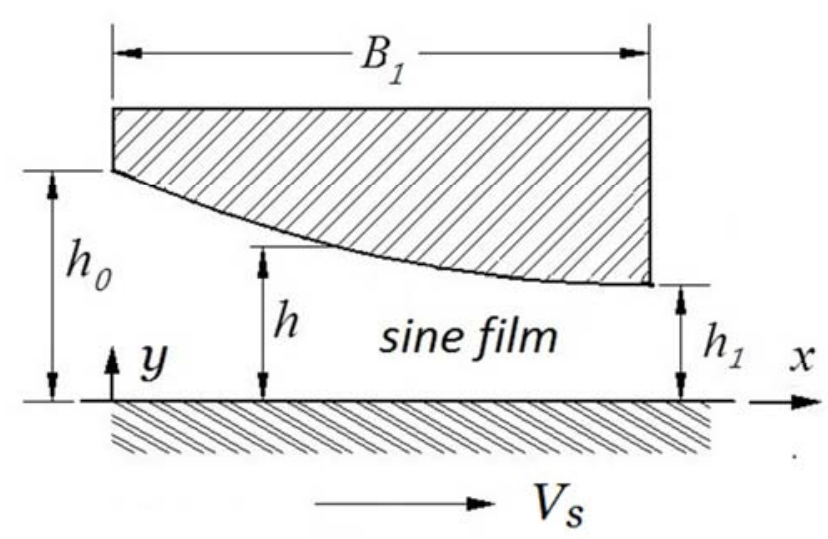

Figure 1. Physical geometry of a sine film thrust bearing lubricated with a non-Newtonian fluid.

The boundary conditions of the velocity in the $x-$ direction are:

$$
\begin{aligned}
& u=V_{s} \text { at } y=0 \\
& u=0 \text { at } y=h
\end{aligned}
$$

Solving equations (1) and equation (5) with the related boundary conditions, one can obtain the expression of the velocity component.

$$
u=u_{A}+u_{B}
$$

where

$$
\begin{gathered}
u_{A}=\frac{1}{\mu}\left[\begin{array}{l}
\frac{\partial p}{\partial x} \frac{y^{2}-h y}{2} \\
+n \cdot\left(\frac{\partial p}{\partial x}\right)^{3}\left(\frac{1}{4} y^{4}-\frac{1}{2} h y^{3}+\frac{3}{8} h^{2} y^{2}-\frac{1}{8} h^{3} y\right)
\end{array}\right] \\
u_{B}=V_{s} \cdot\left\{\begin{array}{l}
1-\frac{y}{h \cdot\left[1+n \cdot(\partial p / \partial x)^{2} h^{2} / 4\right]} \\
-\frac{n \cdot(\partial p / \partial x)^{2}\left(y^{3}-3 h y^{2} / 2+3 h^{2} y / 4\right)}{h \cdot\left[1+n \cdot(\partial p / \partial x)^{2} h^{2} / 4\right]}
\end{array}\right\}
\end{gathered}
$$

The boundary conditions of the velocity in the $y-$ direction are:

$$
\begin{aligned}
& v=0 \text { at } y=0 \\
& v=0 \text { at } y=h
\end{aligned}
$$

The integrated form of the continuity equation (4) across the local film height is

$$
\int_{y=0}^{h} \frac{\partial u}{\partial x} d y+\int_{y=0}^{h} \frac{\partial v}{\partial y} d y=0
$$

Applying the related boundary conditions, one can derive a nonlinear non-Newtonian Reynolds equation for the sine film bearing. 


$$
\frac{d}{d x}\left\{20 h^{3} \frac{d p}{d x}+3 n h^{5}\left(\frac{d p}{d x}\right)^{3}\right\}=120 \mu_{0} V_{s} \frac{d h_{\text {sin }}}{d x}
$$

This Reynolds equation can be used to determine the influences of non-Newtonian rheology on the film pressure and thereafter the load-carrying capacity of the sine film thrust bearing.

In order to conveniently analyze the bearing performances, the non-dimensional variables and parameters are introduced as follows.

$$
\begin{gathered}
X=\frac{x}{B_{1}}, H=\frac{h}{h_{1}}, P=\frac{p h_{1}^{2}}{\mu_{0} V_{s} B_{1}} \\
R=\frac{h_{0}}{h_{1}}, N=\frac{n \mu_{0}^{2} V_{s}^{2}}{h_{1}^{2}} \\
H_{\sin }=\frac{h_{\text {sin }}}{h_{1}}=(R-1) \cdot\left[1-\sin \left(\frac{\pi X}{2}\right)\right]
\end{gathered}
$$

Then the non-dimensional Reynolds equation can be expressed as:

$$
\frac{d}{d X}\left\{20 H^{3} \frac{d P}{d X}+3 N H^{5}\left(\frac{d P}{d X}\right)^{3}\right\}=120 \frac{d H_{\sin }}{d X}
$$

It is difficult to obtain the film pressure from this nonlinear non-Newtonian Reynolds equation. Apply a small perturbation method to the film pressure $P$ operating at small values of the nonlinear non-Newtonian parameter, $-1<<<<+1$, then:

$$
P=P_{0}+N \cdot P_{1}+O\left(N^{2}\right)
$$

Substituting this expansion into the non-dimensional Reynolds equation (19) and collecting the order $O\left(N^{0}\right)$ and the order $O\left(N^{1}\right)$, thereafter one can obtain two coupled equations responsible for the zero order pressure and the first order pressure, respectively.

$$
\begin{aligned}
& \frac{d}{d X}\left\{H^{3} \frac{d P_{0}}{d X}\right\}=-3 \pi(R-1) \cos \left(\frac{\pi X}{2}\right) \\
& \frac{d}{d X}\left\{20 H^{3} \frac{d P_{1}}{d X}+3 N H^{5}\left(\frac{d P_{0}}{d X}\right)^{3}\right\}=0
\end{aligned}
$$

The boundary conditions for the film pressure are:

$$
\begin{aligned}
& P_{0}=0 \text { at } X=0 \\
& P_{1}=0 \text { at } X=0 \\
& P_{0}=0 \text { at } X=1
\end{aligned}
$$

$$
P_{1}=0 \text { at } X=1
$$

Solving these two differential equations, one can obtain

$$
P=\frac{6\left(f_{1} f_{21}-f_{11} f_{2}\right)}{f_{21}}+N \cdot \frac{162\left(f_{31}-f_{3}\right)}{5 f_{21}{ }^{3}}
$$

where

$$
\begin{gathered}
f_{1}(X)=\int_{X=0}^{X} \frac{H_{\text {sin }}}{H^{3}} d X \\
f_{2}(X)=\int_{X=0}^{X} \frac{X}{H^{3}} d X \\
f_{3}(X)=\int_{X=0}^{X} \frac{1}{H^{3}} d X \\
f_{11}=f_{1}(X=1)=\int_{X=0}^{1} \frac{H_{\sin }}{H^{3}} d X \\
f_{21}=f_{2}(X=1)=\int_{X=0}^{1} \frac{X}{H^{3}} d X \\
f_{31}=f_{3}(X=1)=\int_{X=0}^{1} \frac{1}{H^{3}} d X
\end{gathered}
$$

Integrating the film pressure, one can obtain the load-carrying capacity, $w$.

$$
w=\int_{x=0}^{B_{1}} p B_{2} d x
$$

Introduce the non-dimensional load carrying capacity, $W$.

$$
W=\frac{w h_{1}^{2}}{\mu_{0} V_{s} B_{1}^{2} B_{2}}
$$

After performing the integration, one can obtain the non-dimensional load carrying capacity.

$$
W=\frac{6\left(f_{21} \cdot g_{1}-f_{11} \cdot g_{2}\right)}{f_{21}}+N \cdot \frac{162\left(f_{31}-g_{3}\right)}{5 f_{31}{ }^{3}}
$$

where

$$
\begin{aligned}
& g_{1}=\int_{X=0}^{1} f_{1} d X \\
& g_{2}=\int_{X=0}^{1} f_{2} d X \\
& g_{3}=\int_{X=0}^{1} f_{3} d X
\end{aligned}
$$

The values of the non-dimensional load carrying capacity $W$ can be obtained by the method of numerical integration.

The friction force acting at the lower surface is 


$$
f_{f}=-\left.\int_{x=0}^{B_{1}} \tau\right|_{y=0} B_{2} d x
$$

Introduce the non-dimensional variable,

$$
F_{f}=\frac{f_{f} h_{1}}{\mu_{0} V_{s} B_{1} B_{2}}
$$

Then the non-dimensional friction force can be expressed as:

$$
F_{f}=\int_{X=0}^{1}\left\{\begin{array}{l}
\frac{\left.1-N \cdot\left[H^{2}(d P / d X) / 4\right]^{2}\right)}{H} \\
+\frac{H}{2} \frac{d P}{d X}
\end{array}\right\} d X
$$

The friction coefficient is

$$
f_{c}=\frac{-f_{f}}{w}
$$

Applying the non-dimensional friction force and the non-dimensional load capacity, one can obtain the non-dimensional friction parameter $F$.

$$
F=f_{c} \cdot \frac{B_{1}}{h_{1}}=\frac{-F_{f}}{W}
$$

Integrating the velocity field, one can obtain required volume flow rate $q$.

$$
q=\int_{y=0}^{h} u \cdot B_{1} d y
$$

Introduce the non-dimensional required flow rate $Q$.

$$
Q=\frac{q}{V_{s} h_{1} B_{2}}
$$

After performing the integration, one can obtain the non-dimensional required volume flow rate.

$$
Q=\frac{H}{2}-\left[\frac{H^{3}}{12} \frac{d P}{d X}+N \cdot \frac{H^{5}}{80}\left(\frac{d P}{d X}\right)^{3}\right]
$$

\section{Results and Discussion}

In order to present the results, the non-dimensional load capacity are illustrated for the bearing operating at the inlet to outlet film ratio, $R=2 \sim 4.5$ and the non-Newtonian parameter, $N=-0.05 \sim+0.05$.

Figure 2 presents the non-dimensional load capacity $W$ as a function of the inlet-outlet film ratio $R$ for different values of the non-Newtonian parameter $N$. Comparing with the case of Newtonian lubricant $(N=0)$, the influences of non-Newtonian pseudo-plastic fluids $(N=0.05 ; N=0.25)$ reduce the bearing load; however, the effects of non-Newtonian dilatant lubricants $(N=-0.25 ; N=-0.05)$ provide an increase in the load capacity of the sine film thrust bearing.

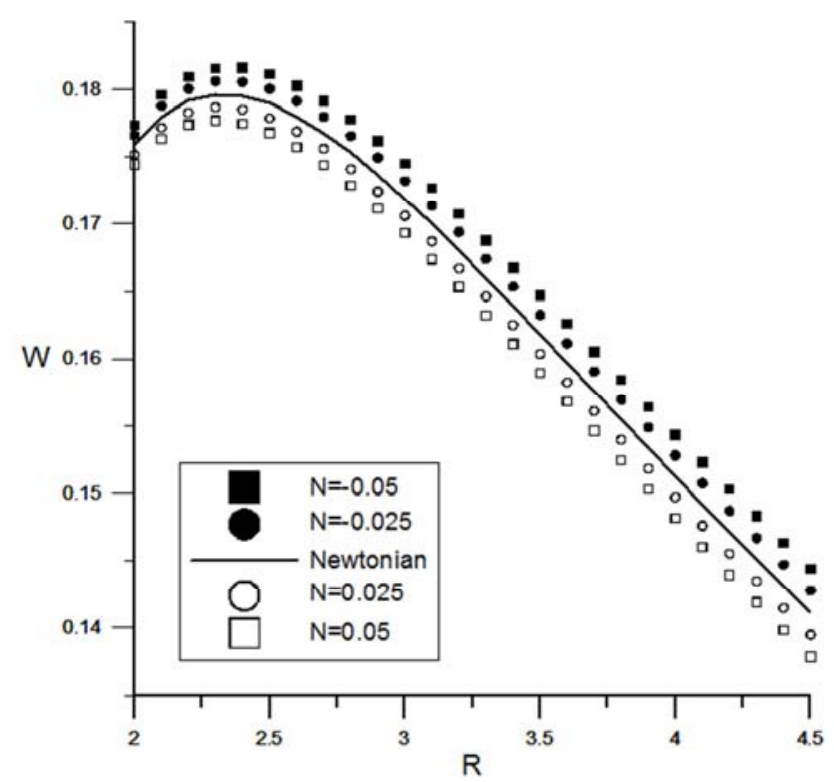

Figure 2. Load capacity $W$ as a function the inlet-outlet film ratio $R$ for different $N$.

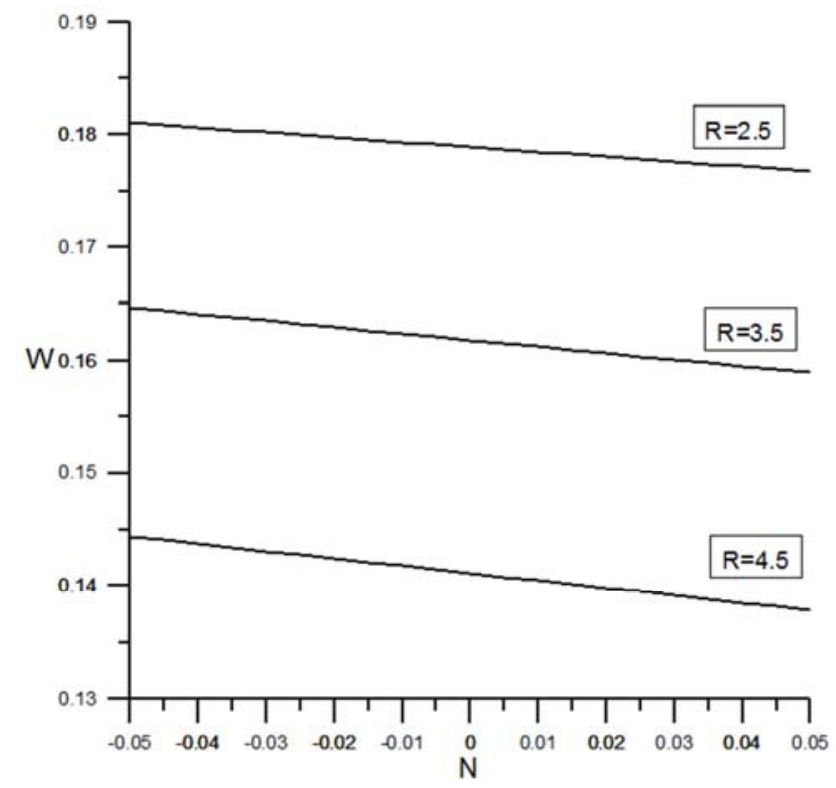

Figure 3. Load capacity $W$ as a function of the non-Newtonian parameter $N$ for different $R$.

Figure 3 shows the non-dimensional load capacity $W$ as a function of the non-Newtonian parameter $N$ for different values of the inlet-outlet film ratio $R$. It is observed that the values of the load capacity $W$ decrease with increasing values of the non-Newtonian parameter $N$ from negative values ( $N<0$, non-Newtonian dilatant lubricants) to zero ( $N=0$, Newtonian lubricants), and then to positive values ( $N>0$, non-Newtonian pseudo-plastic lubricants). 


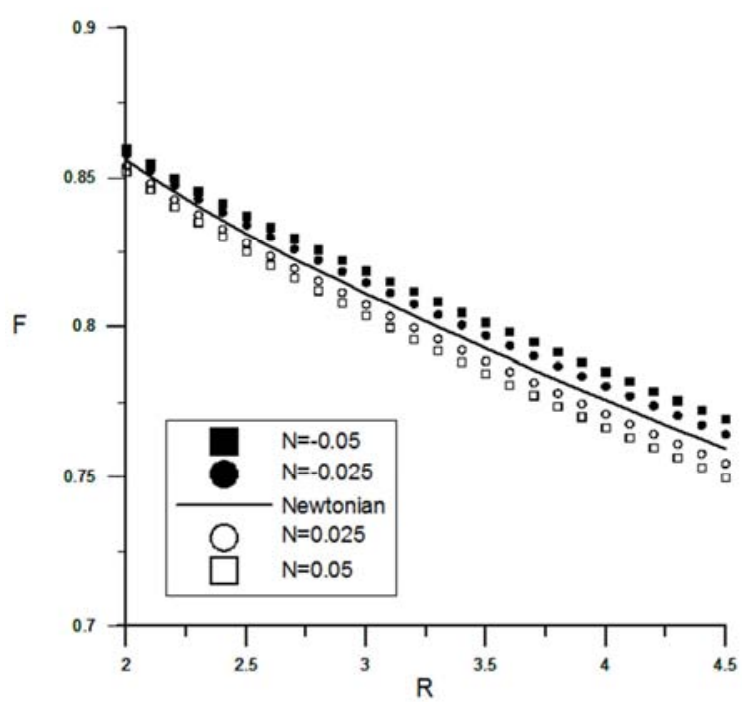

Figure 4. Friction parameter $F$ as a function the inlet-outlet film ratio $R$ for different $N$.

Figure 4 describes the friction parameter $F$ as a function of the inlet-outlet film ratio $R$ for different values of the non-Newtonian parameter $N$. It is observed that the friction parameter decreases with increasing values of the inlet-outlet film ratio. Comparing with the Newtonian-lubricant case ( $N=0$ ), the effects of non-Newtonian pseudo-plastic fluids ( $N=0.05 ; N=0.25$ ) decrease the friction parameter. But, the influences of non-Newtonian dilatant behavior ( $N=-0.25 ; N=-0.05)$ yield higher values of the friction parameter for sine film thrust bearings.

Figure 5 shows the friction parameter $F$ as a function of the non-Newtonian parameter $N$ for different values of the inlet-outlet film ratio $R$. It is also observed that the values of the friction parameter decrease with increasing values of the non-Newtonian parameter from the region of non-Newtonian dilatant lubricants to a Newtonian lubricant, and then to the region of non-Newtonian pseudo-plastic lubricants.

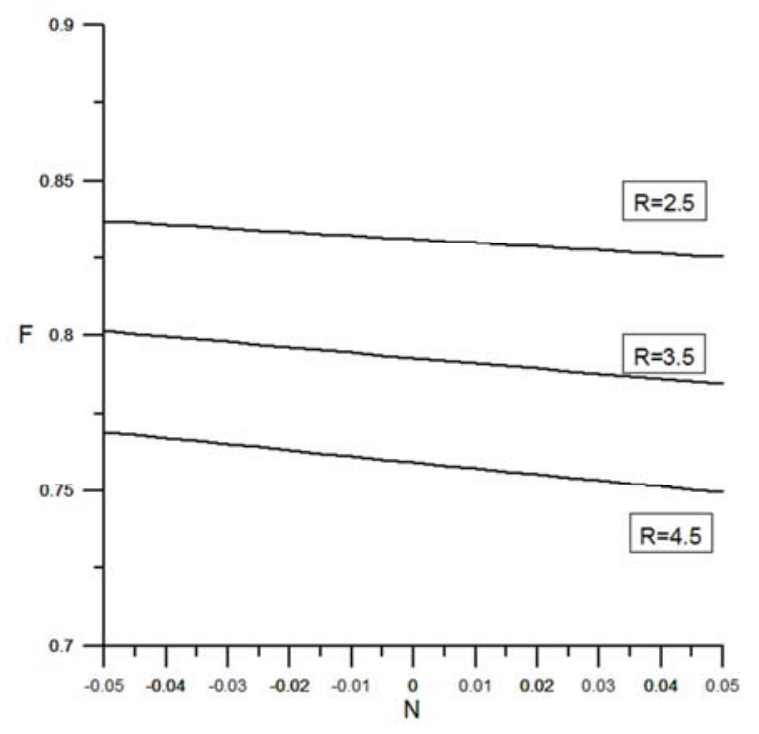

Figure 5. Friction parameter $F$ as a function the non-Newtonian parameter $N$ for different $R$.

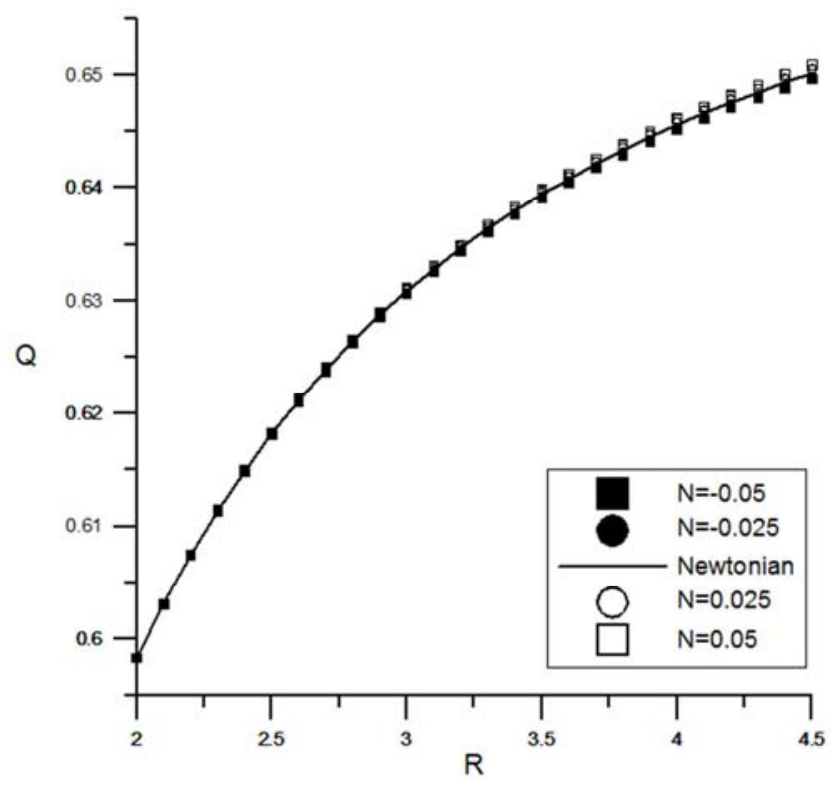

Figure 6. Volume flow rate $Q$ as a function the inlet-outlet film ratio $R$ for different $N$.

Figure 6 depicts the required volume flow rate $Q$ as a function of the inlet-outlet film ratio $R$ for different values of the non-Newtonian parameter $N$. It is observed that the required volume flow rate for sine film thrust bearings increases with increasing values of the inlet-outlet film ratio. It is observed that the influences of non-Newtonian lubricants on the required volume flow rate are slight.

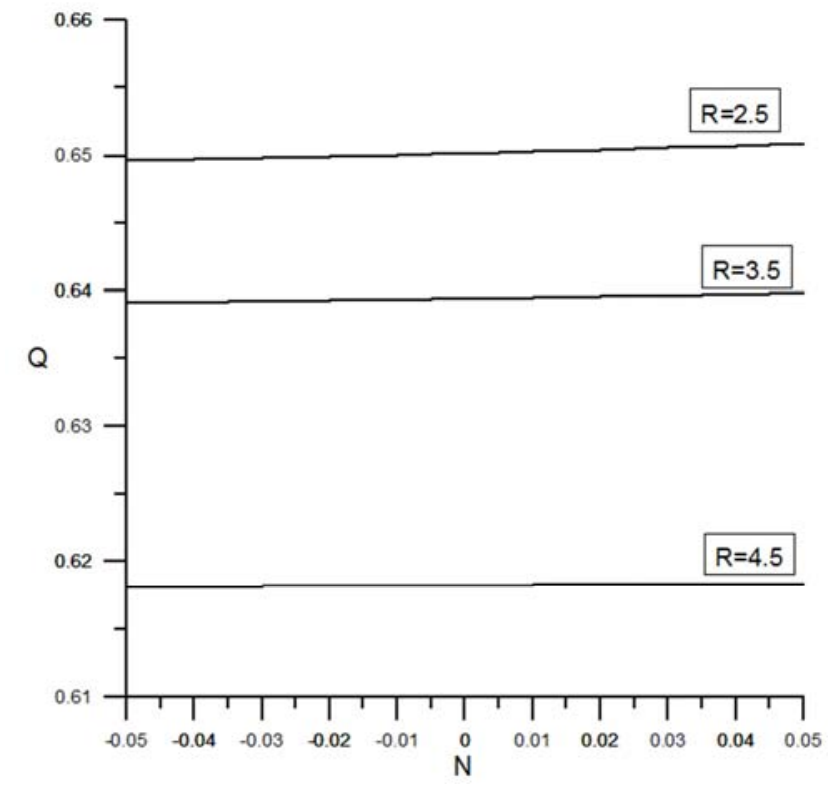

Figure 7. Volume flow rate $Q$ as a function the non-Newtonian parameter $N$ for different $R$.

Figure 7 shows the required volume flow rate $Q$ as a function of the non-Newtonian parameter $N$ for different values of the inlet-outlet film ratio $R$. It is observed that the values of the required volume flow rate increase slightly with increasing values of the non-Newtonian parameter from the region of non-Newtonian dilatant lubricants to a Newtonian 
lubricant, and then to the region of non-Newtonian pseudo-plastic lubricants.

\section{Conclusion}

Taking into account the effects of non-Newtonian rheology, the lubrication performances for a sine film thrust bearing are investigated in the present paper. Based upon the Rabinowitsch fluid model, a nonlinear non-Newtonian Reynolds equation is obtained for a sine film thrust bearing. By employing a small perturbation technique to the nonlinear Reynolds equation, analytical expressions of the film pressure, the load capacity, the friction parameter, and the required volume flow rate are derived. Comparing with the sine film bearing with a Newtonian lubricant, the influences of non-Newtonian pseudo-plastic fluids decrease the load capacity and the friction parameter, and increase the required volume flow rate. However, the effects of non-Newtonian dilatant lubricants provide an increase in the load capacity and the friction parameter, as well as a reduction in the required volume flow rate for the sine film thrust bearings.

\section{References}

[1] C. M. Taylor and D. Dowson, "Turbulent lubrication theory application to design," ASME Journal of Lubrication Technology, 1974, Vol. 96, pp. 36-46.

[2] B. J. Hamrock, Fundamentals of Fluid Film Lubrication. McGraw-Hill: New York, 1994.

[3] J. A. Williams, Engineering Tribology. Oxford University Press Inc.: New York, 1994.

[4] G. Talmage and M. Carpino, "A pseudospectral-finite difference analysis of an infinitely wide slider bearing with thermal and inertia effects," STLE Tribology Transactions, 1997, Vol. 40, pp. 251-258.

[5] J. R. Lin, R. F. Lu, and C. B. Yang, “Linear stability analysis of a wide inclined plane slider bearing," Journal of Science and Technology, 2001, Vol. 10, pp. 349-354.

[6] J. R. Lin and C. R. Hung, "Analysis of dynamic characteristics for wide slider bearings with an exponential film profile," Journal of Marine Science and Technology, 2004, Vol. 12, pp. 217-221.

[7] S. Wada and H. Hayashi, "Hydrodynamic lubrication of journal bearings by pseudoplastic lubricants (Part 2, experimental studies)," Bulletin of the JSME, 1971, Vol. 14, pp. 279-86.

[8] J. R. Lin, "Non-Newtonian squeeze film characteristics between parallel annular disks: Rabinowitsch fluid model," Tribology International, 2012, Vol.52, pp. 190-194.

[9] C. H. Hsu, J. R. Lin, L. J. Mou, and C. C. Kuo, "Squeeze film characteristics of conical bearings operating with non-Newtonian lubricants - Rabinowitsch fluid model," Industrial Lubrication and Tribolog, 2016, Vol. 66, pp. 373-378.

[10] S. C. Sharma, S. C. Jain and P. L. Sah, "Effect of non-Newtonian behaviour of lubricant and bearing flexibility on the performance of slot-entry journal bearing," Tribology International, 2000, Vol. 33, pp. 507-517.

[11] P. Bourging and B. Gay, "Determination of the load capacity of finite width journal bearings by finite element method in the case of a non-Newtonian lubricant," ASME Journal of Tribology, 1984, Vol. 106, pp. 285-290.

[12] J. R. Lin, "Non-Newtonian effects on the dynamic characteristics of one-dimensional slider bearings: Rabinowitsch model," Tribology Letters, 2001, Vol. 10, pp. 237-243. 